\title{
Cell Bonding and Kinships: The stories behind
}

Since the first successful Allogenic Hematopoietic Stem Cell Transplantation (HSCT) in 1968 [1], though thousands of leukaemia patients have been treated worldwide with HSCT, still the clues for engraftment failure following the transplantation remain elusive. The bone marrow mesenchymal or the stromal cells which contribute to the Hematopoietic Stem Cell (HSC) "Niche" and therefore the hematopoiesis and engraftment have a synergy of mutually beneficial enhancement with the HSCs when co-cultured as Kikuchi et al have described in their original research work published in this issue. Multitudes of signalling pathways have been identified that bridge the gap between these two cell populations for a reciprocal relationship and a comprehensive and exhaustive analysis into these mechanisms are important to identify the pathways and molecules which can help improve the engraftment in HSCT. Kikuchi et al have presented an excelled assiduous investigation into the cellular and molecular events in the interactive communication between HSCs and MSCs focussing on the reciprocal upregulation of $\mathrm{NOTCH}$ signalling molecules employing a differentiation-inducible system. The co-culture system they have employed has proven that a reciprocal relationship of mutual enhancement exists that paves way for further exploration of the influence of the MSCs in directing the lineage of the HSCs. In line with this, Puri et al have their work on multifarious potential of Solid Lipid Nanoparticles (SLNs) published in this issue, which discusses on the nanomaterials capability in deciding the fate \& behaviour of mouse mesenchymal stem cells thus adding support to the growing Paradigm of 'Biologists employing Physical and chemical cues to control Cell behaviours'.

Study models for regenerative science have always been on the demand. Xu et al in their article by investigating the characteristics of Feather Follicle Stem Cells (FFSCs) have sprinkled a hope that FFSCs with their extraordinary ability to regenerate and undergo molting cycles can serve as a useful model for studying mechanisms of stem cell differentiation and their involvement in organ regeneration.

The real success of science lies in translating Benchside researches to bedside therapies. This issue has two articles dealing with translatory medicine in Cardiac Diseases, one by Habeler et al drafting their findings on the Direct myocardial implantation of human embryonic stem cells in a dog model of Duchenne cardiomyopathy and another by Gunduz et al communicating their team's experience in experience on cardiac stem cell therapy for Congestive Heart Failure (CHF) in a patient with Hodgkin's lymphoma $(\mathrm{HL})$ who developed $\mathrm{CHF}$ due to ischemic heart disease during the course of lymphoma treatment

This issue also has the Proceedings of the Sixth Annual Plenary Session and Symposium on Regenerative Medicine highlighting abstracts of talks by Experts in the various fields of Stem Cells and Regenerative Medicine

Thus this issue we hope will be a delight to the readers, giving an assorted package of articles dealing with the perspectives of bonding, kinships and signalling mechanisms of cells as the mutually beneficial association among different groups of stem cells add value to each other therefore adding hope to better outcome when clinically translated in challenging situations at the bedside.

Happy reading!!!

[1] Gatti RA, Meuwissen HJ, Allen HD, Hong $\mathrm{R}$, Good RA Immunological reconstitution of sexlinked lymphopenic immunological deficiency. Lancet. 1968 Dec 28;2(7583):1366-9. 